\title{
Physiologically Based Pharmacokinetic Modelling: A Sub-Compartmentalized Model of Tissue Distribution
}

\author{
Max von Kleist* and Wilhelm Huisinga ${ }^{\dagger}$
}

August 10, 2007

\begin{abstract}
We present a sub-compartmentalized model of drug distribution in tissue that extends existing approaches based on the well-stirred tissue model. It is specified in terms of differential equations that explicitly account for the drug concentration in erythrocytes, plasma, interstitial and cellular space. Assuming, in addition, steady state drug distribution and by lumping the different sub-compartments, established models to predict tissue-plasma partition coefficients can be derived in an intriguingly simple way. This direct link is exploited to explicitly construct and parameterize the sub-compartmentalized model for moderate to strong bases, acids, neutrals and zwitterions. The derivation highlights the contributions of the different tissue constituents and provides a simple and transparent framework for the construction of novel tissue distribution models.
\end{abstract}

Keywords: lumped tissue distribution models, partition coefficients, PBPK, $\mathrm{PK} / \mathrm{PD}$, mechanistic modelling, unbound fraction

\section{Introduction}

Physiologically based pharmacokinetic (PBPK) modelling and simulation is increasingly recognized and used as a supporting tool in drug discovery and development (1-6). The aim is to analyze, interpret and predict the pharmacokinetics of drug candidates in the different stages of drug discovery and development $(7,8)$. In toxicokinetics, physiologically based models are frequently used for the assessment of toxicological risks to humans (9-11). A distinctive feature of PBPK models is the underlying mechanistic description of the behavior of drugs in the various tissues and the blood (11). A significant limitation to their application within the pharmaceutical industry has been the requirement of vast quantities of data for model construction (12). In broad terms, PBPK models

\footnotetext{
*Freie Universität Berlin, Department of Mathematics and Computer Science, and DFG Research Center Matheon, Berlin

${ }^{\dagger}$ Hamilton Institute, NUIM, Ireland and DFG Research Center MATHEON, Berlin/Germany, http://www.hamilton.ie/compphysiol
} 
rely on two types of input data: physiological parameters like, e.g., blood flows, organ volumes, tissue composition; and compound-related parameters like, e.g, unbound fraction in plasma, blood-plasma ratio, clearance and tissue partition coefficients.

Partition coefficients are a measure of the steady state drug concentration between two compartments. The tissue-plasma partition coefficient describes the steady state drug distribution in tissue compared to the plasma concentration. The experimental determination of tissue affinities can be costly and time consuming, and requires a substantial amount of compound, which is rarely available during discovery and early candidate selection (12). As a solution to this problem Poulin and Theil proposed in their seminal papers $(13,14)$ to $a$ priori predict the tissue distribution of a drug based on mechanistic description of the underlying physiology and the properties of drugs. Their key idea is to regard the most important tissue constituents - like water, neutral lipids, phospholipids, macro-molecules - and to predict the overall tissue distribution by means of the distribution into these tissue constituents. The extent of drug distribution into the tissue constituents is then estimated from readily available in vitro data. Utilizing these achievements, generic PBPK models have been designed that can be used in early drug discovery $(1-3,15)$. Subsequent predictive models $(12,16,17)$ rely on the same idea, but differ in the way the tissue is composed and/or the distribution into the different constituents is approximated. Some of these models are designed for special compound classes, like moderate to strong bases, acids, zwitterions $(12,16)$.

When aiming at more detailed PBPK models or interfacing with pharmacodynamic processes, current tissue partitioning models have two major limitations: (i) They are built on a lumped tissue compartment model, e.g., lumping together the different tissue sub-compartments, like the interstitial and cellular sub-compartment, and (ii) they assume a quasi-equilibrium between the concentrations in the different sub-compartments ("steady state assumption"). Furthermore, the time-dependence of diffusion processes and/or active transport processes between the different sub-compartments cannot be incorporated, since it violates the underlying steady state assumption.

More detailed and refined PBPK models are known, e.g., (7, 8, 11, 18), but these models are either specifically designed for the compound of interest, or lack a generic parameterization in terms of readily available experimental data. In this article we present a sub-compartmentalized model of drug distribution in tissues, defined in terms of a system of differential equations. These explicitly model the time evolution of the drug concentration in erythrocytes, plasma, the interstitial and cellular space. In the generic case, drug exchange by passive diffusion, non-saturable distribution processes and the absence of metabolic processes is assumed. By exploiting recent developments in a priori prediction of tissue partition coefficients, the sub-compartmentalized tissue distribution model can be parameterized in terms of readily available in vitro data, thus enabling a priori predictions in early drug discovery. Parameterizations for different drug classes, including moderate to strong bases, acids, neutrals and zwitterions, are given. Assuming steady state drug distribution and lumping the different sub-compartments, existing models of tissue partition coefficients can be re-obtained in an intriguingly simple and clear way.

Drug distribution in animals and humans is a complex process, and the presented model is a simplified mechanism-based description of it. In the case 
of partition coefficients, though, such a simplistic description has proven to be very useful in the context of a priori prediction of the pharmacokinetics of new chemical entities. The present model should be seen as a starting point for modelling the tissue distribution of drugs. Whenever addition experimental data or more refined information is available, the model equations should be refined in order to account for the additional insight. This regards transporters in tissues, e.g. (19), distribution in red blood cells, e.g. (20), the possibility of metabolic processes in diverse tissues, e.g. (21), or the consideration of specific tissue binding components that may be important for the specific drug of interest, e.g. (22).

\section{Methods}

Tissue-plasma partition coefficients are a measure of the steady state distribution of the total drug concentration in tissue $C^{\mathrm{t}, \mathrm{ss}}$ compared to the total plasma concentration $C^{\text {p,ss }}$, i.e.,

$$
K^{\mathrm{t}: \mathrm{p}}=\frac{C^{\mathrm{t}, \mathrm{ss}}}{C^{\mathrm{p}, \mathrm{ss}}}
$$

Tissue-unbound plasma partition coefficients $K^{\text {t:up }}$ are a measure of the steady state distribution between the total drug in tissue to the unbound drug in the plasma water $C_{\mathrm{u}}^{\mathrm{p}, \mathrm{ss}}$, i.e.,

$$
K^{\mathrm{t}: \mathrm{up}}=\frac{C^{\mathrm{t}, \mathrm{ss}}}{C_{\mathrm{u}}^{\mathrm{p}, \mathrm{ss}}} .
$$

The vast majority of current generic PBPK models is build on a lumped, wellstirred tissue compartment model (e.g., $(1,23,24))$ using $K^{\text {t:p }}$ or $K^{\text {t:up }}$ as an input parameter.

\section{Sub-compartment tissue distribution}

In this section, we present a sub-compartment tissue distribution model as a generalization of existing models to predict tissue partitioning coefficients. It is specified in terms of differential equations for the drug concentration in erythrocytes (e), plasma (p), interstitial space (i) and cellular space (c), and explicitly allows to model the transfer processes between the different sub-compartments. The choice of sub-compartments is motivated by the availability of physiological data and for pharmacokinetic and pharmacodynamic reasons (11, 19, 20). For the generic parameterization, drug exchange by passive diffusion, non-saturable distribution processes and the absence of metabolic processes is assumed, as in existing models for predicting tissue partition coefficients $(12-14,16)$. Extensions are discussed. Finally, we will demonstrate, how to re-obtain established partition coefficient models by imposing additional assumptions. Vice versa, we will use existing models to construct and a priori parameterize the proposed sub-compartment tissue model. 
Model equations. The sub-compartmentalized tissue distribution model is based on the following assumptions: (i) the distribution of the drug to- and from the organ is governed by advection through the blood flow $Q$ [volume/time]; (ii) the drug exchange between the different sub-compartments of volume $V$ [volume] is governed by passive diffusion, described in terms of the permeabilitysurface area product $P S$ [volume/time]; (iii) only the unbound and unionized drug $C_{\mathrm{un}}=\mathrm{fn} \cdot C_{\mathrm{u}}$ can cross membranes, where $C_{\mathrm{u}}$ denotes the unbound drug concentration in tissue water, and $\mathrm{fn}=C_{\mathrm{un}} / C_{\mathrm{u}}$ denotes the neutral unbound fraction in the water, which is typically calculated according to the HendersonHasselbalch relation. If the compound is not ionizable, we set $\mathrm{fn}=1$. Under these assumptions the time evolution of the total drug concentrations $C$ in the different sub-compartments in a non-eliminating tissue is modeled by the following system of differential equations:

$$
\begin{aligned}
V^{\mathrm{e}} \frac{\mathrm{d}}{\mathrm{d} t} C^{\mathrm{e}}= & Q\left(C_{\mathrm{in}}^{\mathrm{e}}-C^{\mathrm{e}}\right)-P S^{\mathrm{e}: \mathrm{p}}\left(\mathrm{fn}^{\mathrm{e}} C_{\mathrm{u}}^{\mathrm{e}}-\mathrm{fn}^{\mathrm{p}} C_{\mathrm{u}}^{\mathrm{p}}\right) \\
V^{\mathrm{p}} \frac{\mathrm{d}}{\mathrm{d} t} C^{\mathrm{p}}= & Q\left(C_{\mathrm{in}}^{\mathrm{p}}-C^{\mathrm{p}}\right)+P S^{\mathrm{e}: \mathrm{p}}\left(\mathrm{fn}^{\mathrm{e}} C_{\mathrm{u}}^{\mathrm{e}}-\mathrm{fn}^{\mathrm{p}} C_{\mathrm{u}}^{\mathrm{p}}\right) \\
& -P S^{\mathrm{p}: \mathrm{i}}\left(\mathrm{fn}^{\mathrm{p}} C_{\mathrm{u}}^{\mathrm{p}}-\mathrm{fn}^{\mathrm{i}} C_{\mathrm{u}}^{\mathrm{i}}\right) \\
V^{\mathrm{i}} \frac{\mathrm{d}}{\mathrm{d} t} C^{\mathrm{i}}= & P S^{\mathrm{p}: \mathrm{i}}\left(\mathrm{fn}^{\mathrm{p}} C_{\mathrm{u}}^{\mathrm{p}}-\mathrm{fn}^{\mathrm{i}} C_{\mathrm{u}}^{\mathrm{i}}\right)-P S^{\mathrm{i}: \mathrm{c}}\left(\mathrm{fn}^{\mathrm{i}} C_{\mathrm{u}}^{\mathrm{i}}-\mathrm{fn}^{\mathrm{c}} C_{\mathrm{u}}^{\mathrm{c}}\right) \\
V^{\mathrm{c}} \frac{\mathrm{d}}{\mathrm{d} t} C^{\mathrm{c}}= & P S^{\mathrm{i}: \mathrm{c}}\left(\mathrm{fn}^{\mathrm{i}} C_{\mathrm{u}}^{\mathrm{i}}-\mathrm{fn}^{\mathrm{c}} C_{\mathrm{u}}^{\mathrm{c}}\right),
\end{aligned}
$$

where the superscripts $\mathrm{x}=\mathrm{e}, \mathrm{p}, \mathrm{i}, \mathrm{c}$ refer to the different sub-compartments, and the pairs x:y refer to neighboring sub-compartments x and y. $C_{\text {in }}^{\mathrm{p}}$ and $C_{\text {in }}^{\mathrm{e}}$ denote the incoming drug concentrations in plasma and erythrocytes, respectivelytypically the distribution model is part of a whole body physiologically based pharmacokinetic model, in which case the incoming concentrations are linked to out-going concentrations of the preceding organ/tissue (e.g. the arterial concentration). In the above equations, the term $Q\left(C_{\mathrm{in}}^{x}-C^{x}\right)$ with $x=e, p$ models the advection of the drug by the blood flow, while the term $P S^{\mathrm{x}: \mathrm{y}}\left(\mathrm{fn}^{x} C_{\mathrm{u}}^{x}-\mathrm{fn}^{y} C_{\mathrm{u}}^{y}\right)$ with $x, y=e, p, i, c$ models the passive diffusion of the unbound unionized drug across the capillary wall or the cellular membrane.

Under the above assumptions, the distribution of the drug into the different sub-compartments is influenced by three processes: (i) the proportion of unbound to total drug concentration, (ii) the velocity of the transfer processes between sub-compartments, and (iii) the amount of ionization (for ionizable compounds).

In case of a linear, i.e., non-saturable relationship between the unbound aqueous and the total drug concentration, it is

$$
C_{\mathrm{u}}^{\mathrm{x}}=\mathrm{fu}^{\mathrm{x}} \cdot C^{\mathrm{x}},
$$

where $\mathrm{fu}^{x}$ denotes the unbound fraction in the sub-compartment $x$, which is defined as the steady state distribution between the unbound aqueous concentration and the total concentration $\mathrm{fu}^{\mathrm{x}}=C_{\mathrm{u}}^{\mathrm{x}} / C^{\mathrm{x}}$. Replacing $C_{\mathrm{u}}^{\mathrm{x}}$ by $\mathrm{fu}^{\mathrm{x}} \cdot C^{\mathrm{x}}$ results a closed and solvable system of differential equations for the sub-compartment tissue model. If, however, the relation between the total concentration $C$ and the unbound aqueous concentration $C_{\mathrm{u}}$ is assumed to be non-linear, e.g., due to 
saturable binding processes, the generic equations (3)-(6) have to be extended. We will comment on this and illustrate possible extensions below.

First, we consider a linear relation between the unbound aqueous and the total drug concentration. We restrict our consideration to a single sub-compartment $x$ to derive a predictive equation for $\mathrm{fu}^{x}$. Let us assume that the compound partitions into neutral lipids or phospholipids, and is present in water in dissolved form or bound to proteins; other effects are considered neglectable. Then, the total amount of $\operatorname{drug} A^{\mathrm{x}}$ in the sub-compartment $x$ is given by $(12,16,25)$

$$
A^{\mathrm{x}}=A_{\mathrm{u}}+A^{\mathrm{pr}}+A^{\mathrm{nl}}+A^{\mathrm{np}},
$$

where $A_{\mathrm{u}}$ denotes the amount of unbound drug in water, $A^{\mathrm{pr}}$ the amount of bound drug in the water, $A^{\mathrm{nl}}$ and $A^{\mathrm{np}}$ the amount of drug partitioned into neutral lipids (nl) and phospholipids (np). Let us denote by $V^{z: x}$ the fractional volume of the constituent $z$ (e.g., water, neutral or phospholipids) with respect to the total volume of the sub-compartment $x$, i.e.,

$$
V^{z: \mathrm{x}}=\frac{V^{z}}{V^{\mathrm{x}}} .
$$

Dividing eq. (8) by the total sub-compartment volume $V^{x}$ yields

$$
C^{\mathrm{x}}=\left(C_{\mathrm{u}}+C^{\mathrm{pr}}\right) \cdot V^{\mathrm{w}: x}+C^{\mathrm{nl}} \cdot V^{\mathrm{nl}: \mathrm{x}}+C^{\mathrm{np}} \cdot V^{\mathrm{np}: \mathrm{x}}
$$

with $C_{\mathrm{u}}=A_{\mathrm{u}} / V^{\mathrm{w}}, C^{\mathrm{pr}}=A^{\mathrm{pr}} / V^{\mathrm{w}}, C^{\mathrm{nl}}=A^{\mathrm{nl}} / V^{\mathrm{nl}}$, and $C^{\mathrm{np}}=A^{\mathrm{np}} / V^{\mathrm{np}}$. Dividing by the unbound aqueous concentration $C_{\mathrm{u}}$ and exploiting linear binding and distribution processes such that $C_{\mathrm{u}}=\mathrm{fu}^{\mathrm{x}} C^{\mathrm{x}}$, we finally obtain

$$
\frac{1}{\mathrm{fu}^{\mathrm{x}}}=\left(1+\frac{C^{\mathrm{pr}}}{C_{\mathrm{u}}}\right) V^{\mathrm{w}: \mathrm{x}}+\left(\frac{C^{\mathrm{nl}}}{C_{\mathrm{u}}}\right) V^{\mathrm{nl:x}}+\left(\frac{C^{\mathrm{np}}}{C_{\mathrm{u}}}\right) V^{\mathrm{np}: \mathrm{x}} .
$$

The concentration ratios on the right hand side of eq. (11) can be interpreted as partition coefficients associated with the different sub-compartment constituents: the protein-unbound, neutral lipids-unbound and neutral phospholipidsunbound partition coefficients

$$
K^{\mathrm{pr}: \mathrm{u}}=\frac{C^{\mathrm{pr}}}{C_{\mathrm{u}}}, \quad K^{\mathrm{nl}: \mathrm{u}}=\frac{C^{\mathrm{nl}}}{C_{\mathrm{u}}}, \quad K^{\mathrm{np}: \mathrm{u}}=\frac{C^{\mathrm{np}}}{C_{\mathrm{u}}} .
$$

This finally yields

$$
\frac{1}{\mathrm{fu}^{\mathrm{x}}}=\left(1+K^{\mathrm{pr:u}}\right) \cdot V^{\mathrm{w}: \mathrm{x}}+K^{\mathrm{nl:u}} \cdot V^{\mathrm{nl}: \mathrm{x}}+K^{\mathrm{np}: \mathrm{u}} \cdot V^{\mathrm{np}: \mathrm{x}} .
$$

Hence, the inverse of the unbound fraction is a weighted sum of different constituent partition coefficients. The weighting factors are given by the volume fractions of the tissue constituents. The above relation is not restricted to the chosen example, but is much more general: Assume that the sub-compartment comprises water and constituents $z \in\{a, b, c, \ldots\}$. Then, the unbound fraction $\mathrm{fu}^{\mathrm{x}}$ satisfies

$$
\frac{1}{\mathrm{fu}^{\mathrm{x}}}=V^{\mathrm{w}: \mathrm{x}}+\sum_{z \in\{a, b, c, \ldots\}} K^{z: \mathrm{u}} \cdot V^{z: \mathrm{x}} .
$$


In order to derive a priori predictive models, the tissue constituents partition coefficients are approximated by parameters that are assessable through in vitro measurements. To recognize this and to find appropriate experimental realizations was the break-through in a priori determination of partition coefficients $(13,14)$. The differences between existing tissue distribution models regard (i) the tissue constituents that are taken into account and (ii) the approximation of partition coefficients for the resulting constituents by in vitro data. Typically, ionization effects, potential binding to acidic phospholipids and partitioning into the neutral- and phospholipids are processes associated with the cellular space. Important processes in the interstitial space include binding to macro-molecules and ionization effects. Plasma and erythrocytes partitioning are typically measured directly by in vitro experiments.

Finally, let us consider a non-linear relation between the unbound and total concentration. E.g., assume that binding in the interstitial space is saturable, specified in terms of the dissociation constant $K_{\mathrm{D}}$ and the maximum number of possible binding sites $B$. Then, eq. (7) for $x=i$ has to be replaced by the equation

$$
C_{\mathrm{u}}^{\mathrm{i}}=\frac{1}{2}\left(C^{\mathrm{i}}-B-K_{\mathrm{D}}+\sqrt{\left(C^{\mathrm{i}}-B-K_{\mathrm{D}}\right)^{2}+4 K_{\mathrm{D}} C^{\mathrm{i}}}\right) .
$$

Further extensions can be realized in a similar way.

\section{Lumped steady state tissue partitioning}

Common partition coefficient models can be directly derived from the subcompartmentalized tissue distribution model by imposing two additional assumptions: the drug exchange between the different sub-compartments is in dynamical equilibrium (at steady state), and the interstitial and cellular tissue sub-compartments are lumped. From the steady state assumption we deduce that the unionized, unbound aqueous concentration is identical in all subcompartments, i.e.,

$$
\mathrm{fn}^{\mathrm{p}} C_{\mathrm{u}}^{\mathrm{p}}=\mathrm{fn}^{\mathrm{i}} C_{\mathrm{u}}^{\mathrm{i}}=\mathrm{fn}^{\mathrm{c}} C_{\mathrm{u}}^{\mathrm{c}} .
$$

Recalling that the unbound aqueous concentration is defined with respect to water volume and exploiting eq. (16), we obtain for the unbound fraction in the tissue

$$
\begin{aligned}
\frac{1}{\mathrm{fu}^{\mathrm{t}}} & =\frac{C^{\mathrm{t}}}{C_{u}^{\mathrm{t}}}=\frac{\left(V^{\mathrm{i}} C^{\mathrm{i}}+V^{\mathrm{c}} C^{\mathrm{c}}\right) / V^{\mathrm{t}}}{\left(V^{\mathrm{wi}} C_{u}^{\mathrm{i}}+V^{\mathrm{wc}} C_{u}^{\mathrm{c}}\right) / V^{\mathrm{wt}}} \\
& =\left(\frac{V^{\mathrm{i}}}{\mathrm{fn}^{\mathrm{i}} \mathrm{fu}^{\mathrm{i}}}+\frac{V^{\mathrm{c}}}{\mathrm{fn}^{\mathrm{c}} \mathrm{fu}^{\mathrm{c}}}\right)\left(\frac{V^{\mathrm{wi}}}{\mathrm{fn}^{\mathrm{i}}}+\frac{V^{\mathrm{wc}}}{\mathrm{fn}^{\mathrm{c}}}\right)^{-1} \cdot \frac{V^{\mathrm{wt}}}{V^{\mathrm{t}}},
\end{aligned}
$$

where the subscripts wt, wi and wc refer to the tissue-, interstitial- and cellular water, and $V^{\mathrm{t}}=V^{\mathrm{i}}+V^{\mathrm{c}}$ and $V^{\mathrm{wt}}=V^{\mathrm{wi}}+V^{\mathrm{wc}}$. Defining the neutral fraction in tissue $\mathrm{fn}^{\mathrm{t}}$ by

$$
\frac{V^{\mathrm{wt}}}{\mathrm{fn}^{\mathrm{t}}}=\frac{V^{\mathrm{wi}}}{\mathrm{fn}^{\mathrm{i}}}+\frac{V^{\mathrm{wc}}}{\mathrm{fn}^{\mathrm{c}}},
$$

we end up with the relation

$$
\frac{V^{\mathrm{t}}}{\mathrm{fn}^{\mathrm{t}} \mathrm{fu}^{\mathrm{t}}}=\frac{V^{\mathrm{i}}}{\mathrm{fn}^{\mathrm{i}} \mathrm{fu}^{\mathrm{i}}}+\frac{V^{\mathrm{c}}}{\mathrm{fn}^{\mathrm{c}} \mathrm{fu}^{\mathrm{c}}} .
$$


By definition, the tissue-unbound plasma partition coefficient is given by $K^{\text {t:up }}=$ $C^{\mathrm{t}} / C_{\mathrm{u}}^{\mathrm{p}}$. This yields

$$
K^{\mathrm{t}: \mathrm{up}}=\frac{\mathrm{fn}^{\mathrm{p}}}{\mathrm{fn}^{\mathrm{t}}} \cdot \frac{1}{\mathrm{fu}^{\mathrm{t}}},
$$

where we have again exploited that at steady state the unionized, unbound aqueous concentration are identical, i.e., $\mathrm{fn}^{\mathrm{p}} C_{\mathrm{u}}^{\mathrm{p}}=\mathrm{fn}^{\mathrm{t}} C_{\mathrm{u}}^{\mathrm{t}}$. Combining eqs. (20) and (21), we get the central relation between the tissue-unbound plasma partition coefficient and the unbound fractions in the different sub-compartments:

$$
K^{\mathrm{t}: u p}=\frac{\mathrm{fn}^{\mathrm{p}}}{\mathrm{fn}^{\mathrm{i}}} \cdot \frac{V^{\mathrm{i}: \mathrm{t}}}{\mathrm{fu}^{\mathrm{i}}}+\frac{\mathrm{fn}^{\mathrm{p}}}{\mathrm{fn}^{\mathrm{c}}} \cdot \frac{V^{\mathrm{c}: \mathrm{t}}}{\mathrm{fu}^{\mathrm{c}}} .
$$

Since we have $K^{\mathrm{t}: \mathrm{p}}=\mathrm{fu}^{\mathrm{p}} \cdot K^{\mathrm{t}: \mathrm{up}}$, we may alternatively predict the tissue-plasma partition coefficient $K^{\mathrm{t}: \mathrm{p}}$. Finally we remark that eq. (22) easily generalizes to more than two tissue sub-compartments.

\section{Results}

In this section we exploit eqs. (21) and (22) in order to derive and analyze existing models for the prediction of tissue-(unbound) plasma partition coefficients in a unified, transparent and simple way. On the other hand, the design principles and the parameterization of the existing models can be used to construct a priori sub-compartment tissue distribution models.

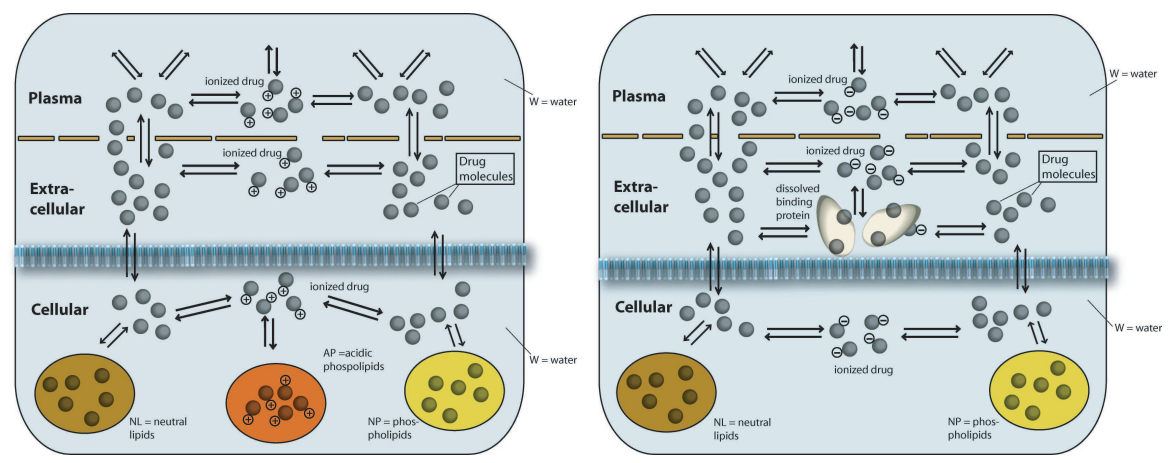

Figure 1: Tissue decomposition and processes underlying the a priori partition coefficient models for moderate to strong bases by Rodgers et al. (left), and for neutrals and acids by Rodgers and Rowland (right). For details, see the text.

\section{Moderate to strong bases and type 1 zwitterions}

Rodgers et al. $(12,16)$ recently developed mechanistic equations to predict the tissue-unbound plasma partition coefficient for moderate to strong bases $\left(\mathrm{pK}_{\mathrm{a}} \geq 7.0\right)$ and type 1 zwitterions (at least one $\mathrm{pK}_{\mathrm{a}} \geq 7.0$ ). The model assumes that the unbound (dissolved) drug is possibly ionized in the extra-cellular and intra-cellular space. In the intra-cellular space, the ionized drug may bind to acidic phospholipids (rem), while the neutral form may distribute into neutral lipids (nl) and phospholipids (np). Furthermore it is assumed that only 
the neutral species can cross membranes by passive diffusion; see Fig. 1, left for illustration. In the following derivation, we identify the extra-cellular and intra-cellular space in $(12,16)$ with the interstitial and cellular space. Moreover, we denote the water in the interstitial and cellular space by (wi) and (wc).

The model is based on the following approximations of the constituent partition coefficients: (i) cellular residual tissue components-unbound drug partitioning:

$$
K^{\mathrm{rem}: \mathrm{uc}}=\left(1-\mathrm{fn}^{\mathrm{c}}\right) K_{\mathrm{A}, \mathrm{AP}}\left[\mathrm{AP}^{-}\right]^{\mathrm{rem}}
$$

where $\left[\mathrm{AP}^{-}\right]^{\mathrm{rem}}$ denotes the concentration of acidic phospholipids in the residual space, with corresponding association constant $K_{\mathrm{A}, \mathrm{AP}}$. (ii) Neutral lipidsunbound drug partitioning:

$$
K^{\mathrm{nl}: \mathrm{uc}}=\mathrm{fn}^{\mathrm{c}} P_{*: \mathrm{w}}
$$

where $P_{*: \mathrm{w}}$ is chosen to be the octanol-water partition coefficient $P_{\mathrm{o}: \mathrm{w}}$ for nonadipose tissue and the vegetable oil-water partition coefficient $P_{\mathrm{vo} \text { : }}$ for adipose tissue. (iii) Neutral phospholipids-unbound drug partitioning:

$$
K^{\mathrm{np}: \mathrm{uc}}=\mathrm{fn}^{\mathrm{c}}\left(0.3 \cdot P_{*: \mathrm{w}}+0.7\right)
$$

assuming that neutral phospholipids behave like a mixture of $30 \%$ neutral lipids and $70 \%$ water (as initially suggested by Poulin and Theil in (13)).

Sub-compartmentalized tissue distribution model. Since no interstitial binding is considered, the unbound fraction in the interstitial space is

$$
\frac{1}{f u^{\mathrm{i}}}=1
$$

while we obtain

$$
\begin{aligned}
\frac{1}{\mathrm{fu}^{\mathrm{c}}}= & V^{\mathrm{wc}: \mathrm{c}}+\mathrm{fn}^{\mathrm{c}} P_{*: \mathrm{w}} V^{\mathrm{nl}: \mathrm{c}}+\mathrm{fn}^{\mathrm{c}}\left(0.3 P_{*: \mathrm{w}}+0.7\right) V^{\mathrm{np}: \mathrm{c}}+ \\
& \left(1-\mathrm{fn}^{\mathrm{c}}\right) K_{\mathrm{A}, \mathrm{AP}}\left[\mathrm{AP}^{-}\right]^{\mathrm{c}}
\end{aligned}
$$

for the cellular unbound fraction, where we exploited the relation $\left[\mathrm{AP}^{-}\right]^{\mathrm{c}}=$ $\left[\mathrm{AP}^{-}\right]^{\mathrm{rem}} V^{\mathrm{rem}: \mathrm{c}}$. Note that $V^{x: \mathrm{c}}=V^{x: \mathrm{t}} \cdot V^{\mathrm{t}} / V^{\mathrm{c}}$, so that volume fractions with reference to the cellular space can easily be converted into those with reference to the tissue space (and thus we can use the readily available data in $(12,16,18)$ ).

Lumped steady state model. Exploiting eq. (22) we obtain the tissueunbound plasma partition coefficient for moderate to strong bases:

$$
\begin{aligned}
K^{\mathrm{t}: \mathrm{up}=} & V^{\mathrm{wi}: \mathrm{t}}+\frac{\mathrm{fn}^{\mathrm{p}}}{\mathrm{fn}^{\mathrm{c}}} V^{\mathrm{wc}: \mathrm{t}}+\mathrm{fn}^{\mathrm{p}} P_{*: \mathrm{w}} V^{\mathrm{nl}: \mathrm{t}}+\mathrm{fn}^{\mathrm{p}}\left(0.3 P_{*: \mathrm{w}}+0.7\right) V^{\mathrm{np}: \mathrm{t}}+ \\
& \mathrm{fn}^{\mathrm{p}} \frac{1-\mathrm{fn}^{\mathrm{c}}}{\mathrm{fn}^{\mathrm{c}}} K_{\mathrm{A}, \mathrm{AP}}\left[\mathrm{AP}^{-}\right]^{\mathrm{t}}
\end{aligned}
$$


where $\left[\mathrm{AP}^{-}\right]^{\mathrm{t}}$ denotes the concentration of acidic phospholipids in tissue, which is related to the corresponding cellular concentration $\left[\mathrm{AP}^{-}\right]^{\mathrm{c}}$ by $\left[\mathrm{AP}^{-}\right]^{\mathrm{c}} V^{\mathrm{c}: \mathrm{t}}=$ $\left[\mathrm{AP}^{-}\right]^{\mathrm{t}}$. We remark that

$$
\frac{1-\mathrm{fn}^{\mathrm{c}}}{\mathrm{fn}^{\mathrm{c}}}=10^{-\left(\mathrm{pH}_{\mathrm{c}}-\mathrm{pK}_{\mathrm{a}}\right)}
$$

for mono-protonic bases. Typically, values for $K_{\mathrm{A}, \mathrm{AP}}$ are not readily available for the different tissues. In order to estimate the unknown association constants, Rodgers et al. suggested to determine $K_{\mathrm{A}, \mathrm{AP}}$ for the erythrocytes from the blood-plasma ratio $\mathrm{B}: \mathrm{P}$ and use this value as an approximation for the association constants in the other tissues. For details see (12, 26). Parameter values can be found in $(12,16)$ for the species rat.

\section{Very weak bases, neutrals, acids and type 2 zwitterions}

In (16) Rodgers and Rowland developed mechanistic equations to predict tissueunbound plasma partition coefficients for very weak bases $\left(\mathrm{pK}_{\mathrm{a}}<7.0\right)$, neutrals, acids and type 2 zwitterions (no $\mathrm{pK}_{\mathrm{a}} \geq 7.0$ ). The model assumes that the drug is dissolved and possibly ionized (for acids, very weak bases, and type 2 zwitterions) in the extra-cellular and intra-cellular space. It may bind in either form macromolecules (albumin for acids and weak bases, lipoproteins for neutrals) in the extra-cellular space, and distribute into neutral lipids and phospholipids in unionized form in the intra-cellular space. Furthermore it is assumed that only the neutral species can passively diffuse across membranes; see Fig. 1 (right) for illustration. In the following derivation, we again identify the extra-cellular and intra-cellular space in (16) with the interstitial and cellular space.

The model is based on the following approximations of the constituents partition coefficients: (i) Interstitial protein-unbound drug partitioning:

$$
K^{\text {pr:ui }}=\mathrm{K}_{\mathrm{A}, \mathrm{PR}} \mathrm{PR}^{\mathrm{wi}}
$$

where $\mathrm{PR}^{\mathrm{wi}}$ denotes the concentration of interstitial binding protein (albumin in the case of acidic, very weak basic and type 2 zwitterions, or lipoproteins in the case of neutral compounds), and $\mathrm{K}_{\mathrm{A}, \mathrm{PR}}$ refers to the corresponding association constant. (ii) Neutral lipids-unbound drug partitioning:

$$
K^{\mathrm{nl}: \mathrm{uc}}=\mathrm{fn}^{\mathrm{c}} P_{*: \mathrm{w}},
$$

where $P_{*: \mathrm{w}}$ is chosen to be the octanol-water partition coefficient $P_{\mathrm{o}: \mathrm{w}}$ for nonadipose tissue and the vegetable oil-water partition coefficient $P_{\mathrm{vo} \text { :w }}$ for adipose tissue. (iii) Neutral phospholipids-unbound drug partitioning:

$$
K^{\mathrm{np}: \mathrm{uc}}=\mathrm{fn}^{\mathrm{c}}\left(0.3 P_{*: \mathrm{w}}+0.7\right)
$$

assuming that neutral phospholipids behave like a mixture of $30 \%$ neutral lipids and $70 \%$ water (as above). 
Sub-compartmentalized tissue distribution model. For the unbound fraction in the interstitial space, eq. (14) yields

$$
\frac{1}{\mathrm{fu}^{\mathrm{i}}}=1+\mathrm{K}_{\mathrm{A}, \mathrm{PR}} \mathrm{PR}^{\mathrm{i}}
$$

while we obtain

$$
\frac{1}{\mathrm{fu}^{\mathrm{c}}}=V^{\mathrm{wc}: \mathrm{c}}+\mathrm{fn}^{\mathrm{c}} P_{*: \mathrm{w}} V^{\mathrm{nl}: \mathrm{c}}+\mathrm{fn}^{\mathrm{c}}\left(0.3 P_{*: \mathrm{w}}+0.7\right) V^{\mathrm{np}: \mathrm{c}}
$$

for the cellular unbound fraction.

Lumped steady state model. Exploiting eq. (22) we obtain the tissueunbound plasma partition coefficient for very weak bases, acids, neutrals and group 2 zwitterions

$$
\begin{aligned}
K^{\mathrm{t}: \mathrm{up}}= & V^{\mathrm{i}: \mathrm{t}}+\mathrm{K}_{\mathrm{A}, \mathrm{PR}} \mathrm{PR}^{\mathrm{t}}+\frac{\mathrm{fn}^{\mathrm{p}}}{\mathrm{fn}^{\mathrm{c}}} V^{\mathrm{wc}: \mathrm{t}}+\mathrm{fn}^{\mathrm{p}} P_{*: \mathrm{w}} V^{\mathrm{nl}: \mathrm{t}} \\
& +\mathrm{fn}^{\mathrm{p}}\left(0.3 P_{*: \mathrm{w}}+0.7\right) V^{\mathrm{np}: \mathrm{t}}
\end{aligned}
$$

where we have exploited that $\mathrm{PR}^{\mathrm{t}}=\mathrm{PR}^{\mathrm{i}} V^{\mathrm{i} t \mathrm{t}}$. Rodgers and Rowland suggest to determine $\mathrm{K}_{\mathrm{A}, \mathrm{PR}}$ in plasma from $\mathrm{fu}^{\mathrm{p}}$ and $P_{\mathrm{o} \text { : }}$. For details see (16, eq. (13)). Parameter values can be found in $(16,26)$ for the species rat.

\section{Poulin-Theil model}

\section{homogeneous model}

Poulin and Theil model

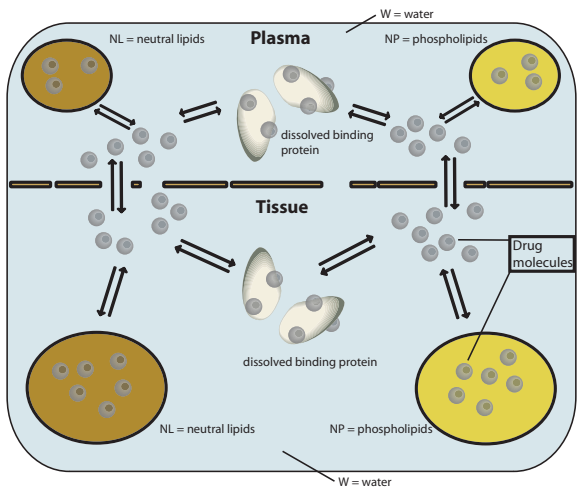

Willmann et al. model

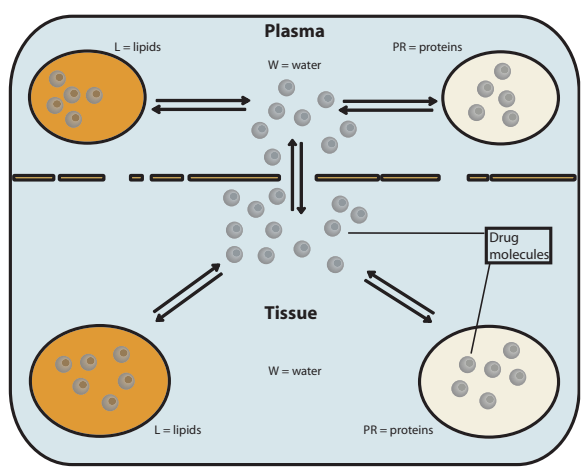

Figure 2: Tissue decomposition and processes underlying the a priori partition coefficient models by Poulin and Theil (left), and by Willmann et al. (right). For details, see the text.

In their seminal papers in 2000/01 (13, 14), Poulin and Theil proposed an in silico approach to a priori predict tissue-plasma partition coefficients solely based on few compound specific in vitro data. They assumed that the compound is present in dissolved form in tissue water, that it may bind to macromolecules 
in the interstitial space, and distribute into neutral lipids or phospholipids in the cellular space, while other effects are considered negligible. These equations have subsequently been corrected by Berezhovskiy (25, eq. (64)) (see also (16)). For the present model we will only derive the unbound fraction in the tissue $\mathrm{fu}^{\mathrm{t}}$, since only the total tissue water is considered by Poulin and Theil rather than a distinction between the interstitial and cellular water as in the previous models. The unbound fraction in tissue is linked to the tissue partition coefficient via $K^{\mathrm{t}: \mathrm{p}}=\mathrm{fu}^{\mathrm{p}} / \mathrm{fu}^{\mathrm{t}}$, if ionization is not considered. The lumping of the tissue space in the model by Poulin and Theil has the following consequence for the sub-compartmentalized tissue model: Either the generic equations are reduced to account for the change of tissue concentration only, or alternatively, one sets $f u^{i}=f u^{c}=f u^{t}$ and stays with the full system of equations.

The model (including the corrections) is based on the following approximations: (i) Protein-unbound drug partitioning:

$$
K^{\text {pr:ut }}= \begin{cases}1 /\left(2 \mathrm{fu}^{\mathrm{p}}\right)-1 / 2 ; & \text { non-adipose tissue } \\ 0 ; & \text { adipose tissue } \\ 1 / \mathrm{fu}^{\mathrm{p}}-1 ; & \text { plasma }\end{cases}
$$

where $\mathrm{fu}^{\mathrm{p}}$ denotes the unbound fraction in plasma as measured by in vitro assays. (ii) Neutral lipids-unbound drug partitioning:

$$
K^{\text {nl:ut }}=P_{*: \mathrm{w}}
$$

where $P_{*: \mathrm{w}}$ is chosen to be the octanol-water partition coefficient $P_{\mathrm{o}: \mathrm{w}}$ for nonadipose tissue and the vegetable oil-water partition coefficient $P_{\text {vo:w }}$ for adipose tissue. (iii) Neutral phospholipids-unbound drug partitioning:

$$
K^{\text {np:ut }}=0.3 \cdot K^{\text {nl:ut }}+0.7,
$$

where it is assumed that neutral phospholipids behave like a mixture of $30 \%$ neutral lipids and $70 \%$ water. For ionizable compounds, $P_{\text {vo:w }}$ has to be replaced by $\mathrm{fn} \cdot P_{\mathrm{vo}: \mathrm{w}}$. In view of eq. (38) and (39) this implies that a correction for ionizable compounds is only made for adipose tissue (see Discussion).

Sub-compartmentalized tissue distribution model. Based on the fundamental relation (13), the unbound fraction in non-adipose tissue $\mathrm{fu}^{\mathrm{t}}$ is given by

$$
\frac{1}{\mathrm{fu}^{\mathrm{t}}}=\left(\frac{1}{2 \mathrm{fu}^{\mathrm{p}}}+\frac{1}{2}\right) V^{\mathrm{wt}: \mathrm{t}}+P_{\mathrm{o}: \mathrm{w}} V^{\mathrm{nl}: \mathrm{t}}+\left(0.3 P_{\mathrm{o}: \mathrm{w}}+0.7\right) V^{\mathrm{np}: \mathrm{t}}
$$

For adipose tissue it is

$$
\frac{1}{\mathrm{fu}^{\mathrm{t}}}=V^{\mathrm{wt}: \mathrm{t}}+P_{\mathrm{vo}: \mathrm{w}} V^{\mathrm{nl}: \mathrm{t}}+\left(0.3 P_{\mathrm{vo}: \mathrm{w}}+0.7\right) V^{\mathrm{np}: \mathrm{t}}
$$

Lumped steady state model. By neglecting ionization ( $\mathrm{fn}=1$ ), as in (13), we exploit eq. $(21)$ and $K^{\mathrm{t}: \mathrm{p}}=K^{\mathrm{t}: \mathrm{up}} \cdot \mathrm{fu}^{\mathrm{p}}$ to obtain the tissue-plasma partition coefficient as published by Poulin and Theil, with subsequent corrections 
according to Berezhovskiy. For non-adipose tissue it is

$$
K^{\mathrm{t}: \mathrm{p}}=\frac{\left(1 /\left(2 \mathrm{fu}^{\mathrm{p}}\right)+1 / 2\right) V^{\mathrm{w}: \mathrm{t}}+P_{\mathrm{o}: \mathrm{w}} V^{\mathrm{nl}: \mathrm{t}}+\left(0.3 P_{\mathrm{o}: \mathrm{w}}+0.7\right) V^{\mathrm{np}: \mathrm{t}}}{\left(1 / \mathrm{fu}^{\mathrm{p}}\right) V^{\mathrm{w}: \mathrm{p}}+P_{\mathrm{o}: \mathrm{w}} V^{\mathrm{nl}: \mathrm{p}}+\left(0.3 P_{\mathrm{o}: \mathrm{w}}+0.7\right) V^{\mathrm{np}: \mathrm{p}}},
$$

and for adipose tissue it is

$$
K^{\mathrm{t}: \mathrm{p}}=\frac{V^{\mathrm{w}: \mathrm{t}}+P_{\mathrm{vo}: \mathrm{w}} V^{\mathrm{nl}: \mathrm{t}}+\left(0.3 P_{\mathrm{vo:w}}+0.7\right) V^{\mathrm{np}: \mathrm{t}}}{\left(1 / \mathrm{fu}^{\mathrm{p}}\right) V^{\mathrm{w}: \mathrm{p}}+P_{\mathrm{vo}: \mathrm{w}} V^{\mathrm{nl}: \mathrm{p}}+\left(0.3 P_{\mathrm{vo}: \mathrm{w}}+0.7\right) V^{\mathrm{np}: \mathrm{p}}},
$$

where $V^{\mathrm{w}: \mathrm{p}}, V^{\mathrm{nl}: \mathrm{p}}, V^{\mathrm{np}: \mathrm{p}}$ are the respective fractions of water, neutral lipids and phospholipids in plasma. Parameter values can be found in $(13,27)$ for the species rabbit, rat, mouse and human.

\section{Willmann et al. model}

In (17), Willmann et al. proposed an alternative mechanistic model for the calculation of the tissue-plasma partition coefficient $K^{\mathrm{t}: \mathrm{p}}$. In contrast to the previous models Willmann et al. use the membrane affinity to quantify binding to lipids. It is assumed that the compound can dissolve in tissue water, bind to proteins (pr) and membrane lipids (l). In distinction to the previous models, Willmann et al. consider the proteins as a separate phase.

The model is based on the following approximations (17): (i) Protein-unbound drug partitioning:

$$
K^{\text {pr:ut }}= \begin{cases}\mathrm{PR} / K_{\mathrm{D}} ; & \text { plasma } \\ 10^{-5} \mathrm{MA} ; & \text { tissue }\end{cases}
$$

where PR denotes the concentration of albumin in the blood plasma, and $K_{\mathrm{D}}$ denotes the dissociation constant for serum proteins. (ii) Lipids-unbound drug partitioning:

$$
K^{\text {l:ut }}=\mathrm{MA}
$$

where MA denotes the membrane affinity. For ionizable compounds, the tissue protein-unbound drug partitioning is corrected with the ionization constant $\mathrm{pK}_{\mathrm{a}}$ yielding

$$
K^{\text {pr:ut }}=10^{-5}\left(9.9 \frac{1}{1+\left(8 / \mathrm{pK}_{\mathrm{a}}\right)^{18}}+0.1\right) \mathrm{MA} .
$$

Sub-compartmentalized tissue distribution model. Based on the fundamental relation (13), the unbound fraction in tissue $\mathrm{fu}^{\mathrm{t}}$ is given by

$$
\frac{1}{\mathrm{fu}^{\mathrm{t}}}=V^{\mathrm{wt}: \mathrm{t}}+K^{\mathrm{pr}: \mathrm{wt}} V^{\mathrm{pr}: \mathrm{t}}+\mathrm{MA} \cdot V^{\mathrm{l}: \mathrm{t}} .
$$


Lumped steady state model. Exploiting eq. $(21)$ and $K^{\mathrm{t}: \mathrm{p}}=K^{\mathrm{t}: \mathrm{up}} \cdot \mathrm{fu}^{\mathrm{p}}$ we obtain the tissue-plasma partition coefficient

$$
K^{\mathrm{t}: \mathrm{p}}=\frac{V^{\mathrm{w}: \mathrm{t}}+K^{\mathrm{pr}: \mathrm{ut}} V^{\mathrm{pr}: \mathrm{t}}+\mathrm{MA} \cdot V^{\mathrm{l}: \mathrm{t}}}{V^{\mathrm{w}: \mathrm{p}}+K^{\mathrm{pr}: \mathrm{up}} V^{\mathrm{pr}: \mathrm{p}}+\mathrm{MA} \cdot V^{\mathrm{l}: \mathrm{p}}}
$$

Parameter values can be found in (28).

\section{Parameterization of the sub-compartmentalized tissue dis- tribution model}

We have demonstrated, how existing approaches to a priori predict tissue(unbound) plasma partition coefficients can be exploited to predict the unbound fractions in the interstitial and cellular space. In our context, these values can readily be used to parameterize the sub-compartment tissue model. This allows us to establish different parameterization for different drug classes. Beyond $\mathrm{fu}^{\mathrm{i}}$ and $\mathrm{fu}^{\mathrm{c}}$, the unbound fractions in plasma and erythrocytes, as well as permeability-surface area products and the different volumes of the subcompartments are needed.

The unbound fraction in plasma $\mathrm{fu}^{\mathrm{p}}$ is directly determined by in vitro measurements, while the unbound fraction in erythrocytes $\mathrm{fu}^{\mathrm{e}}$ can be derived from the blood-plasma ratio $\mathrm{B}: \mathrm{P}$ according to

$$
\frac{1}{f u^{e}}=\frac{B: P-(1-\text { hct })}{\text { hct } \cdot f u^{p}} \cdot \frac{f n^{e}}{f n^{p}},
$$

where hct denotes the hematocrit value. A detailed summary on red blood cell partitioning can be found in (20). The unbound fractions can be used with eq. (7) to parameterize the sub-compartment model. A permeability-surface area product $P S$ is obviously not present in the lumped steady state models, so we have to adopt a different approach. In the early stage of drug discovery, the permeability-surface area product may well be chosen identically to a sufficiently large value in order to account for a fast and instantaneous exchange. In this case our model mimics the dynamical equilibrium (comparable to the partition coefficient models). However, it still allows to directly access the concentrations in the different sub-compartments and to include active transport processes. Once experimental data is available, it might be possible to estimate the permeability-surface area product, cf. e.g, (18). However, a generic parameterization for a large class of drugs is not known.

Typically, tissue volume is regarded as extra-vascular space, comprising the interstitial and the cellular space, while the organ space includes the vascular space in addition to the interstitial and cellular space. Tissue volume data can be found in the literature, see e.g., $(1,12,16,29)$. In (18, Table A2), values for the vascular volume fractions $V^{\mathrm{v} \text { :org }}$ and for the interstitial volume fractions $V^{\text {i:org }}$ of diverse organs have been published for rats and assumed to be identical for humans. Utilizing the hematocrit (hct) value, we may thus compute all necessary volumes for the sub-compartmentalized tissue distribution model: $V^{\text {t:org }}=1-V^{\mathrm{v} \text { :org }}, V^{\mathrm{v}}=V^{\mathrm{v} \text { :org }} \cdot V^{\mathrm{t}} / V^{\mathrm{t}: \text { org }}, V^{\mathrm{e}}=$ hct $\cdot V^{\mathrm{v}}, V^{\mathrm{p}}=(1-$ hct $) \cdot V^{\mathrm{v}}$, $V^{\mathrm{i}}=V^{\mathrm{i} \text { org }} \cdot V^{\mathrm{t}} / V^{\mathrm{t}: \text { org }}, V^{\mathrm{c}}=\left(1-V^{\mathrm{v} \text { :org }}-V^{\mathrm{i} \text { org }}\right) \cdot V^{\mathrm{t}} / V^{\mathrm{t}: \text { org }}$. 


\section{Extensions of the sub-compartmentalized tissue distribu- tion model}

The main advantage of the sub-compartmentalized tissue distribution model is the ability to extent and refine it. As a simple example, we consider the integration of binding to a target $B$ in the interstitial space. In this example we assume a non-saturable binding to macro-molecules (proteins) specified in terms of $\mathrm{fu}_{\mathrm{pr}}$, and a saturable binding to a target protein $B$ with dissociation constant $K_{\mathrm{D}}$. The model refinement can easily be realized by adding evolution equations for the drug concentration that is bound to macro-molecules $C_{\mathrm{pr}}^{\mathrm{i}}$, for the concentration that is bound to the target proteins $C_{\text {target }}^{\mathrm{i}}$, and the free target protein concentration $B_{u}$ to eqs. (3)-(6):

$$
\begin{aligned}
V^{\mathrm{i}} \frac{\mathrm{d}}{\mathrm{d} t} C_{\mathrm{pr}}^{\mathrm{i}} & =V^{\mathrm{i}} \cdot k_{\mathrm{fast}}\left(\frac{1-\mathrm{fu}_{\mathrm{pr}}}{\mathrm{fu}_{\mathrm{pr}}} \cdot C_{u}^{\mathrm{i}}-C_{\mathrm{pr}}^{\mathrm{i}}\right) \\
V^{\mathrm{i}} \frac{\mathrm{d}}{\mathrm{d} t} C_{\text {target }}^{\mathrm{i}} & =V^{\mathrm{i}} \cdot k_{\text {fast }}\left(\frac{C_{u}^{\mathrm{i}} B_{u}}{K_{\mathrm{D}}}-C_{\text {target }}^{\mathrm{c}}\right) \\
V^{\mathrm{i}} \frac{\mathrm{d}}{\mathrm{d} t} B_{u} & =V^{\mathrm{i}} \cdot k_{\text {fast }}\left(\left(B_{\mathrm{tot}}-B_{u}\right)-\frac{C_{u}^{\mathrm{i}} B_{u}}{K_{\mathrm{D}}}\right)
\end{aligned}
$$

where the unbound concentrations equals $C_{\mathrm{u}}^{\mathrm{i}}=C^{\mathrm{i}}-C_{\mathrm{pr}}^{\mathrm{i}}-C_{\mathrm{target}}^{\mathrm{i}}$. The parameter $k_{\text {fast }}\left[1 /\right.$ time] denotes a large rate constant, while $B_{\text {tot }}$ denotes the total target protein concentration in the interstitial space. In the case of the equations above, it is also possible to derive an algebraic expression for $C_{\mathrm{u}}^{\mathrm{i}}(\mathrm{cf} .(18))$ :

$$
\begin{aligned}
C_{\mathrm{u}}^{\mathrm{i}}= & \frac{1}{2} \mathrm{fu}_{\mathrm{pr}}\left(\left(C^{\mathrm{i}}-K_{\mathrm{D}} / \mathrm{fu}_{\mathrm{pr}}-B\right)\right. \\
& \left.+\sqrt{\left(C^{\mathrm{i}}-K_{\mathrm{D}} / \mathrm{fu}_{\mathrm{pr}}-B\right)^{2}+4 C^{\mathrm{i}} K_{\mathrm{D}} / \mathrm{fu}_{\mathrm{pr}}}\right),
\end{aligned}
$$

which would substitute eq. (7) with $x=i$. Along the same lines it is possible to include active transport processes between different sub-compartments, or to integrate entire signalling pathways in order to account for drug related effects.

\section{Discussion}

A major challenge in the construction of a priori tissue distribution models is the choice of the considered tissue constituents - like macro-molecules, neutral lipids etc. - as well as the way the resulting constituent partition coefficients are approximated by in vitro data. The presented approach highlights these two points and offers a flexible and transparent way to design novel tissue distribution models.

Common tissue distribution coefficients can be easily derived by assuming steady state drug distribution and by lumping the different sub-compartments. In comparison to the derivations in the original papers $(12-14,16,17)$, the relation in eq. (22) is intriguingly simple and may serve as a way to develop novel partitioning coefficient models in the future. Within this setting, common features as well as the differences between the existing models can easily be assessed. All considered models regard protein binding and distribution into water and lipids as major processes determining the distribution of the drug into the 
tissue. While Willmann et al. regard tissue lipids as a whole, Poulin and Theil as well as Rodgers et al. further distinguish between neutral and phospholipids. In addition, different in vitro parameters are used to approximate the resulting constituent partition coefficients ( $\log \mathrm{P}$ vs. MA). A major difference between the models is the way they take ionization effects into account. Rodgers et al. model ionization based on the Henderson-Hasselbalch relation. In contrast, Willmann et al. propose an empirical model, cf. eq. (46), that has been optimized based on experimental data. In the Poulin and Theil model there are some inconsistencies regarding ionization. Only the vegetable oil-water partition coefficient is corrected for ionization, accounting for a modified neutral lipids-unbound drug partitioning. However, the neutral phospholipids-unbound drug partition coefficient in eq. $(38)$ is not correctly modified $\left(\left(0.3 \mathrm{fn} \cdot K^{\text {nl:ut }}+0.7\right)\right.$ instead of $\left.\mathrm{fn} \cdot\left(0.3 K^{\mathrm{nl}: \mathrm{ut}}+0.7\right)\right)$. In addition, ionization in non-adipose tissue is not accounted for. Another difference between the models is the way protein binding is modelled. Willmann et al. use the membrane affinity (MA) to estimate protein binding in tissue, while Poulin and Theil as well as Rodgers et al. rely on the fraction unbound in plasma $\left(f u^{p}\right)$. While in the Rodgers et al. models $f^{p}$ is appropriately modified in order to account for distribution processes into e.g. neutral lipids and phospholipids within the plasma, this is not realized in the Poulin and Theil model.

Eqs. (3)-(6) model the distribution of a drug into a non-eliminating tissue/organ. For eliminating tissues/organs, the equations have to extended to account for the metabolic processes. The advantage of a description in terms of different equations is that such processes can easily be integrated, in particular for two and more, possible saturable processes. In the case of eliminating tissues, eq. (22) is not valid anymore, since the underlying assumption eq. (16) is violated. In this case, tissue partition coefficients can be directly determined from the concentrations in the different sub-compartments, i.e., $K^{\mathrm{t}: \mathrm{p}}=C^{\mathrm{t}} / C^{\mathrm{p}}=\left(V^{\mathrm{i}} C^{\mathrm{i}}+V^{\mathrm{c}} C^{\mathrm{c}}\right) /\left(V^{\mathrm{i}}+V^{\mathrm{c}}\right) / C^{\mathrm{p}}$. In special cases, also the partition coefficient can be adapted to account for elimination, see (25, eq. (55)) for non-saturable tissue elimination.

In PBPK modelling, the most widely used model of tissue distribution is the well-stirred or perfusion rate-limited model $(5,11)$. It lumps together the extravascular space of the tissue and is build on a single tissue compartment. The idea of decomposing organs/tissues into blood, interstitial and cellular space was proposed earlier (e.g.,(11)), but refined models have not been used in a generic way due to the increased amount of parameters needed. The presented approach directly addresses this parameterization problem. A major advantage of the sub-compartmentalized distribution model is its capability to link tissue distribution to effect related signalling pathways or metabolic/gene regulatory networks. This becomes increasingly important in view of the current modelling and simulation needs in the pharmaceutical industry. As outlined in (34), failure due to pharmacodynamic and toxicological issues is of major concern. To be supportive, physiologically based pharmacokinetic models should provide interfaces for pharmacodynamic or toxicological models (19).

The sub-compartmentalized tissue distribution model is of increased complexity in comparison to the common well-stirred lumped tissue model, which results in additional differential equations to be solved. Taking explicitly the four sub-compartments erythrocyte, plasma, interstitium and cellular space into account quadruples the number of equations; considering a lumped vascular 
space would still result in a threefold number of equations. From a numerical point of view, the number of equations is still relatively small compared to applications, e.g., in chemical engineering, automobile or aviation industry, and efficient numerical algorithms to solve (stiff) problems are available $(30,31)$. However, when aiming at analyzing the overall model or at estimating parameters, the increased complexity can become a problem. Here the relation between the detailed sub-compartment model and the lumped model can be exploited to reduce the complexity, and further reduction techniques may be applicable (cf., e.g., $(32,33))$.

In this article, we have focussed on a generic parameterization of the subcompartment distribution model in terms of readily available in vitro data. We utilized recent developments in $(12,13,17)$ to establish predictive models for small-molecular compounds. For the class of biologics, it can also be crucial to incorporate cellular processes in order to correctly account for the distribution processes (like, e.g., internalization processes in the case of monoclonal antibodies). The equations (3)-(6) can easily be extended, e.g. to account for active transport processes $(19,20)$, linear- or nonlinear metabolism $(20,21)$, binding to other relevant molecules (20, 22), including target molecules (19) and downstream effects. This opens the door for a wide range of applications in pharmacokinetics and -dynamics.

Acknowledgements It is a pleasure to thank Andreas Reichel, Philip Lienau and Reinhard Nubbemeyer (Bayer Schering Pharma) for fruitful discussions, and Stefan Willmann (Bayer Technology Services) for comments on the partition coefficient model (17). Financial support by the DFG Research Center MATHEON "Mathematics for key technologies: Modelling, simulation, and optimization of real-world processes", Berlin is acknowledged.

\section{References}

1. P. Poulin and F. P. Theil. Prediction of pharmacokinetics prior to in vivo studies. II. Generic physiologically based pharmacokinetic models of drug disposition. J Pharm Sci, 91:1358-70, 2002.

2. F. P. Theil, T. W. Guentert, S. Haddad and P. Poulin. Utility of physiologically based pharmacokinetic models to drug development and rational drug discovery candidate selection. Toxicol Letters, 138:29-49, 2003.

3. H. M. Jones, N. Parrott, K. Jorga and T. Lavé. A novel strategy for physiologically based predictions of human pharmacokinetics. Clin Pharmacokinet, 45:511-42, 2006.

4. H. van de Waterbeemd and E. Gifford. ADMET in silico modelling: towards prediction paradise? Nat Rev Drug Discov, 2:192-204, 2003.

5. G. M. Grass and P. J. Sinko. Physiologically-based pharmacokinetic simulation modelling. Adv Drug Deliv Rev, 54:433-51, 2002. 
6. N. Parrott, N. Paquereau, P. Coassolo and T. Lavé. An evaluation of the utility of physiologically based models of pharmacokinetics in early drug discovery. J Pharm Sci, 94:2327-2343, 2005.

7. I. Nestorov. Whole body pharmacokinetic models. Clin. Pharmacokinet, 42 :883-908, 2003.

8. Y. Kwon. Handbook of Essential Pharmacokinetics, Pharmacodynamics and Drug metabolism for Industrial Scientists. Kluwer Academic/Plenum Publishers, New York, 2001.

9. M. Reddy, R. S. Yang, M. E. Andersen, H. J. Clewell. Physiologically Based Pharmacokinetic Modeling Wiley, Hoboken, 2005

10. M.E. Andersen. Development of physiologically based pharmacokinetic and physiologically based pharmacodynamic models for applications in toxicology and risk assessment. Toxicol. Lett., 79:35-44, 1995

11. L. E. Gerlowski and R. K. Jain. Physiologically based pharmacokinetic modeling: principles and applications. J Pharm Sci, 72:1103-1127, 1983.

12. T. Rodgers, D. Leahy and M. Rowland. Physiologically based pharmacokinetic modeling 1: predicting the tissue distribution of moderate-to-strong bases. J Pharm Sci, 94:1259-1276, 2005.

13. P. Poulin and F. P. Theil. A priori prediction of tissue:plasma partition coefficients of drugs to facilitate the use of physiologically-based pharmacokinetic models in drug discovery. J Pharm Sci, 89:16-35, 2000.

14. P. Poulin, K. Schoenlein and F. P. Theil. Prediction of adipose tissue: plasma partition coefficients for structurally unrelated drugs. J Pharm Sci, 90:436-447, 2001.

15. C. Lüpfert and A. Reichel. Development and application of physiologically based pharmacokinetic-modeling tools to support drug discovery. Chem Biodivers, 2:1462-1486, 2005.

16. T. Rodgers and M. Rowland. Physiologically based pharmacokinetic modelling 2: predicting the tissue distribution of acids, very weak bases, neutrals and zwitterions. J Pharm Sci, 95:1238-1257, 2006.

17. S. Willmann, J. Lippert and W. Schmitt. From physicochemistry to absorption and distribution: predictive mechanistic modelling and computational tools. Expert Opin Drug Metab Toxicol, 1:159-68, 2005.

18. R. Kawai, D. Mathew, C. Tanaka and M. Rowland. Physiologically based pharmacokinetics of cyclosporine A: extension to tissue distribution kinetics in rats and scale-up to human. J Pharmacol Exp Ther, 287:457-68, 1998.

19. J. H. Lin. Tissue distribution and pharmacodynamics: a complicated relationship. Curr Drug Metab, 7:39-65, 2006.

20. P. H. Hinderling. Red blood cells: a neglected compartment in pharmacokinetics and pharmacodynamics. Pharmacol Rev, 49:279-295, 1997. 
21. X. Ding and L. S. Kaminsky. Human extrahepatic cytochromes P450: function in xenobiotic metabolism and tissue-selective chemical toxicity in the respiratory and gastrointestinal tracts. Annu Rev Pharmacol Toxicol, 43: $149-173,2003$

22. B. Fichtl, A. V. Nieciecki and K. Walter. Tissue binding versus plasma binding of drugs: general principles and pharmacokinetic consequences. Adv Drug Res, 20:118-166, 1991.

23. H. H. Chow. A physiologically based pharmacokinetic model of zidovudine (azt) in the mouse: model development and scale-up to humans. J Pharm Sci, 86:1223-1228, 1997.

24. O. Sugita, Y. Sawada, Y. Sugiyama, T. Iga and M. Hanano. Physiologically based pharmacokinetics of drug-drug interaction: a study of tolbutamidesulfonamide interaction in rats. J Pharmacokinet Biopharm, 10:297-316, 1982.

25. L. M. Berezhkovskiy. Volume of distribution at steady state for a linear pharmacokinetic system with peripheral elimination. J Pharm Sci, 93:162840, 2004.

26. T. Rodgers and M. Rowland. Mechanistic approaches to volume of distribution predictions: understanding the processes. Pharm Res, 24:918-933, 2007.

27. P. Poulin and F. P. Theil. Prediction of pharmacokinetics prior to in vivo studies. 1. Mechanism-based prediction of volume of distribution. J Pharm Sci, 91:129-56, 2002.

28. J. Keldenich. A physiological/mechanistic model for predicting organ/plasma partitioning and volume of distribution. LogP2004 The 3rd Lipophilicity Symposium, 2004.

29. R. P. Brown, M. D. Delp, S. L. Lindstedt, L. R. Rhomberg and R. P. Beliles. Physiological parameter values for physiologically based pharmacokinetic models. Toxicol Ind Health, 13:407-84, 1997.

30. P. Deuflhard and F. Bornemann. Scientific computing with ordinary differential equations. Springer, New York, 2002.

31. E. Hairer and G. Wanner. Solving ordinary differential equations: Stiff and differential-algebraic problems. Springer, New York, 1996.

32. I. A. Nestorov, L. J. Aarons, P. A. Arundel and M. Rowland. Lumping of whole-body physiologically based pharmacokinetic models. J Pharmacokinet Biopharm, 26:21-46, 1998.

33. C. Brochot, J. Toth and F. Y. Bois. Lumping in pharmacokinetics. $J$ Pharmacokinet Pharmacodyn, 32:719-736, 2005.

34. I. Kola and J. Landis. Can the pharmaceutical industry reduce attrition rates? Nat Rev Drug Discov, 3:711-5, 2004. 